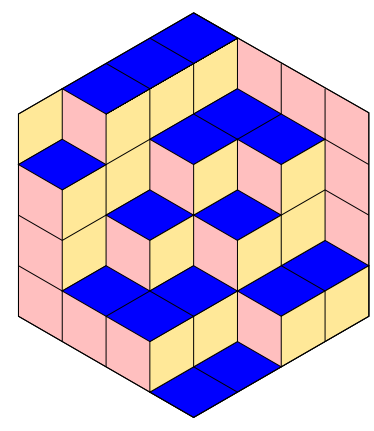

Matjaž Konvalinka, Robin Sulzgruber \& Vasu Tewari

Trimming the permutahedron to extend the parking space

Volume 4, issue 4 (2021), p. 663-674.

<http://alco.centre-mersenne.org/item/ALCO_2021_4_4_663_0>

(c) The journal and the authors, 2021.

Some rights reserved.

(c) BY This article is licensed under the

Creative Commons ATtribution 4.0 InTERnational License.

http://creativecommons.org/licenses/by/4.0/

Access to articles published by the journal Algebraic Combinatorics on the website http://alco.centre-mersenne.org/ implies agreement with the Terms of Use (http://alco.centre-mersenne.org/legal/).

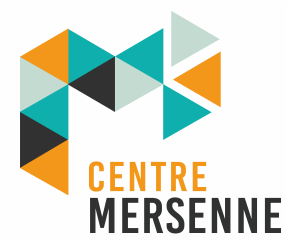

Algebraic Combinatorics is member of the Centre Mersenne for Open Scientific Publishing www.centre-mersenne.org 


\title{
Trimming the permutahedron to extend the parking space
}

\author{
Matjaž Konvalinka, Robin Sulzgruber \& Vasu Tewari
}

\begin{abstract}
Berget and Rhoades asked whether the permutation representation obtained by the action of $S_{n-1}$ on parking functions of length $n-1$ can be extended to a permutation action of $S_{n}$. We answer this question in the affirmative. We realize our module in two different ways. The first description involves binary Lyndon words and the second involves the action of the symmetric group on the lattice points of the trimmed standard permutahedron.
\end{abstract}

\section{INTRODUCTION}

Recall that a parking function of length $n-1$ is a sequence of nonnegative integers $\left(a_{1}, \ldots, a_{n-1}\right)$ whose nondecreasing rearrangement $\left(b_{1}, \ldots, b_{n-1}\right)$ satisfies $b_{i} \leqslant i-1$. It is immediate that the set of parking functions of length $n-1$ carries a permutation action of $S_{n-1}$. This parking function representation, henceforth referred to as Park $_{n-1}$, was first studied by Haiman in his influential work [10]. In their study of an extension of Park ${ }_{n-1}$, Berget and Rhoades asked [5, Section 4] whether the permutation action of $S_{n-1}$ on parking functions of length $n-1$ could be extended to a permutation action of $S_{n}$. It is worth remarking that the study of "hidden" actions of a larger symmetric group on spaces where a smaller symmetric group acts naturally has received a fair amount of attention in recent years; see for instance [7, 8, 9, 13, 20]. It is in this broader context that our work is set.

The extension $V_{n-1}$ in [5] is realized by considering the $\mathbb{C}$-span of a distinguished set of polynomials in $n$ variables first studied by Postnikov and Shapiro [15]. While the aforementioned set of polynomials is $S_{n}$-stable, it does not form a basis for $V_{n-1}$ in general. Berget and Rhoades work with a basis for $V_{n-1}$ that is not $S_{n}$-stable. To establish that the restriction of $V_{n-1}$ from $S_{n}$ to $S_{n-1}$ is indeed Haiman's Park ${ }_{n-1}$, Berget and Rhoades use Gröbner-theoretic techniques to construct a linear subspace of $V_{n-1}$ with an $S_{n-1}$-stable monomial basis indexed by parking functions.

Our point of departure is a particular permutahedron in $\mathbb{R}^{n}$ whose set of lattice points is equinumerous with the set of parking functions on length $n-1$, thereby providing a plausible candidate. Given a tuple $\lambda=\left(\lambda_{1} \geqslant \cdots \geqslant \lambda_{n}\right)$, we define the permutahedron $P_{\lambda} \subset \mathbb{R}^{n}$ to be the convex hull of the $S_{n}$ orbit of $\lambda$. We denote the set of lattice points $P_{\lambda} \cap \mathbb{Z}^{n}$ by $\operatorname{Lat}\left(P_{\lambda}\right)$. For $n \geqslant 2$, define $\delta_{n}$ to be the partition

Manuscript received 28th April 2020, revised 24th March 2021, accepted 25th March 2021.

KEYwORDS. $h$-positivity, Lyndon word, parking function, permutahedron.

ACKNowledgements. The first author acknowledges the financial support from the Slovenian Research Agency (research core funding No. P1-0294). 
$(n-2, \ldots, 1,0,0)$. It is clear that $S_{n}$ acts on $\operatorname{Lat}\left(P_{\delta_{n}}\right)$. Let $\gamma_{n}$ denote the associated representation. Here is our main result which answers the question posed by Berget and Rhoades.

THEOREM 1.1. We have that

$$
\operatorname{Res}_{S_{n-1}}^{S_{n}}\left(\gamma_{n}\right)=\operatorname{Park}_{n-1}
$$

We refer the reader to Example 4.1 for the explicit computation in the case $n=4$. Thus Theorem 1.1 tells us that $\gamma_{n}$ is a permutation representation which extends the parking function representation. Furthermore, a conjecture of the first and third author [11, Conjecture 3.1] may be restated as claiming that $\gamma_{n}$ is isomorphic to the ungraded Berget-Rhoades representation $V_{n-1}$.

Our approach is indirect and builds off of earlier work [11] by the first and third author wherein a family of $S_{n}$-representations $\widehat{\mathrm{PF}}_{n, c}$ that restrict to Park ${ }_{n-1}$ is constructed. For an appropriately chosen value of $c$, this representation is isomorphic to what we consider here. We give an explicit $h$-positive expansion for the Frobenius characteristic of this representation in terms of binary Lyndon words satisfying a straightforward constraint. Our proof goes via an intermediate module $\mathcal{C}_{m, n}$ that we analyze in depth as well. The representation $\gamma_{n}$ is obtained by identifying elements of $\mathcal{C}_{1, n}$ up to a natural equivalence relation. Finally, we can compute the character of $\gamma_{n}$ by appealing to [11, Theorem 3.2]. This does not appear to be a straightforward task from the definition of $\gamma_{n}$. The character values allow us to make a connection with recent work of Ardila, Schindler and Vindas-Meléndez [2], which we state next.

Corollary 1.2. Let $\Pi_{n}$ denote the standard permutahedron $P_{(n-1, \ldots, 1,0)}$. Given $\sigma \in$ $S_{n}$ with cycle type $\left(\lambda_{1}, \ldots, \lambda_{\ell}\right)$, let $\Pi_{n}^{\sigma}$ denote the set of points in $\Pi_{n}$ that are fixed by $\sigma$. Suppose $\operatorname{GCD}\left(\lambda_{1}, \ldots, \lambda_{\ell}\right)=1$. Then the normalized volume of $\Pi_{n}^{\sigma}$ is equal to the number of lattice points in $P_{\delta_{n}}$ fixed by $\sigma$.

If $\sigma$ is the identity permutation, then Corollary 1.2 says that normalized volume of the standard permutahedron in $\mathbb{R}^{n}$ is equal to the number of lattice points in $P_{\delta_{n}}$. The former is well known to equal $n^{n-2}$ [18]. Thus, in this specific instance, our result reduces to a special case of $[14$, Corollary 11.5].

For maximum generality, we work in the setting of rational parking functions for the majority of this paper. In Section 4, we specialize to arrive at Theorem 1.1.

\section{THE SETUP}

To keep our exposition brief, we refer the reader [19, Chapter 7] for all notions pertaining to the combinatorics of symmetric functions which are not defined explicitly here.

2.1. The modules $\mathcal{C}_{m, n}$ AND $\widehat{\mathcal{C}}_{m, n}$. Given positive integers $m$ and $n$, set $N:=m n$ and

$$
c_{m, n}:=\frac{(N-2)(n-1)}{2} .
$$

This given, consider the set of $N$-tuples defined as follows:

$$
\mathcal{C}_{m, n}:=\left\{\left(x_{1}, \ldots, x_{N}\right) \mid 0 \leqslant x_{i} \leqslant n-1, \sum_{1 \leqslant i \leqslant N} x_{i}=c_{m, n}(\bmod n)\right\} .
$$

Clearly, $\left|\mathcal{C}_{m, n}\right|=n^{N-1}$. Geometrically, one may interpret $C_{m, n}$ to be the set of lattice points in the cube $[0, n-1]^{N}$ in $\mathbb{R}^{n}$ that lie on certain translates of the hyperplane $x_{1}+\cdots+x_{N}=0$. Note that $S_{N}$ acts on $\mathcal{C}_{m, n}$ by permuting coordinates and we denote 
the resulting permutation action by $\tau_{m, n}$. We abuse notation and use $\mathcal{C}_{m, n}$ to denote both the set and the resulting $S_{N}$-module.

Let $\Lambda_{k}$ denote the set of tuples $\lambda=\left(\lambda_{1} \geqslant \cdots \geqslant \lambda_{k}\right)$ in $\mathbb{N}^{k}$. Here $\mathbb{N}$ denotes the set of nonnegative integers. We refer to elements of $\Lambda_{k}$ as partitions. Given $\left(\lambda_{1}, \ldots, \lambda_{k}\right) \in$ $\Lambda_{k}$, we refer to $\lambda_{i}$ 's as the parts of $\lambda$. In particular, we consider 0 to be a part. Given any sequence $\mathbf{x}=\left(x_{1}, \ldots, x_{k}\right) \in \mathbb{N}^{k}$, we define $\operatorname{sort}(\mathbf{x})$ to be the partition obtained by sorting $\mathbf{x}$ in nonincreasing order. Let

$$
Y_{m, n}:=\Lambda_{N} \cap \mathcal{C}_{m, n}
$$

Clearly, elements of $Y_{m, n}$ index the orbits of $\mathcal{C}_{m, n}$ under $\tau_{m, n}$.

By drawing $\lambda \in Y_{m, n}$ as a Young diagram in French notation so that the lower left corner coincides with the origin in $\mathbb{Z}^{2}$, we may identify $\lambda$ with a lattice path $L_{\lambda}$ that starts at $(n, 0)$, ends at $(0, N)$, and takes vertical and horizontal steps of unit length. All coordinates here are Cartesian. It will be convenient to extend $L_{\lambda}$ to an infinite path $L_{\lambda}^{\infty}$ by repeating $L_{\lambda}$. Figure 1 depicts $\lambda=(2,2,1,1,0) \in Y_{1,5}$. The shaded region represents the $5 \times 5$ box where $\lambda$ is drawn, the red path depicts a fragment of $L_{\lambda}^{\infty}$, and the thickened subpath represents $L_{\lambda}$.

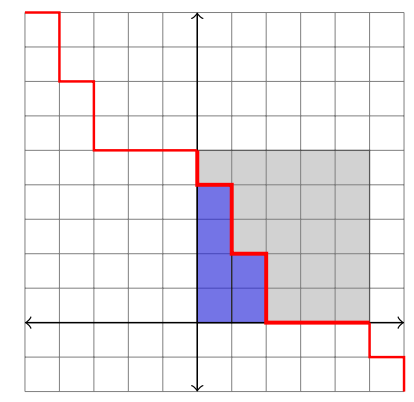

Figure 1. Path corresponding to $\lambda=(2,2,1,1,0)$.

We define the shift map shift mapping $\{0, \ldots, n-1\}^{N}$ to itself via the rule

$$
\operatorname{shift}\left(x_{1}, \ldots, x_{N}\right):=\left(x_{1}+1, \ldots, x_{N}+1\right),
$$

where addition is performed modulo $n$. It is clear that shift ${ }^{n}$ is the identity map. This map allows us to define an equivalence relation $\sim$ on $\mathcal{C}_{m, n}$ by declaring two sequences to be equivalent if one is obtained by applying shift ${ }^{j}$ to the other for some $j \in \mathbb{N}$. Since the sum of the coordinates remains invariant modulo $n$ upon applying the shift map, our $S_{N}$ action on $\mathcal{C}_{m, n}$ descends to an action $\widehat{\tau}_{m, n}$ on the set of equivalences classes $\mathcal{C}_{m, n} / \sim$. We denote this set (and the associated $S_{N}$ module) by $\widehat{\mathcal{C}}_{m, n}$. Since every equivalence class has $n$ elements, we have that $\left|\widehat{\mathcal{C}}_{m, n}\right|=n^{N-2}$.

REMARK 2.1. The careful reader should note that our construction works equally well with $c_{m, n}$ replaced by any integer. Thus, one obtains a family of $S_{N}$ modules in this manner. The analog of the set $\widehat{\mathcal{C}}_{m, n}$ has the property that every equivalence class therein has a unique element $\left(x_{1}, \ldots, x_{N}\right)$ so that $\left(x_{1}, \ldots, x_{N-1}\right)$ is a rational parking function. See discussion in [11, Section 5] to this end. It follows that the modules under consideration in this article are a special case of those studied in loc. cit., the choice $c_{m, n}$ having been made to answer the question of Berget and Rhoades. This choice is not merely a fortuitous coincidence as $c_{m, n}$ is closely related to the area statistic on parking functions, and the latter already plays a role in [5]. The one pertinent upshot of this discussion is that $\widehat{\mathcal{C}}_{1, n}$ restricts to $\operatorname{Park}_{n-1}$. 
2.2. BINARY WORDS AND $Y_{m, n}$. We now interpret the partitions in $Y_{m, n}$ as certain words in the alphabet $\{0,1\}$ as this will shed more light into their structure. By reading $L_{\lambda}$ from right to left and recording a 0 (respectively 1 ) for each horizontal (respectively vertical) step, we obtain a word $w_{\lambda}$ of length $(m+1) n$ in $\{0,1\}$. Clearly, $w_{\lambda}$ begins with a 0 and has $m n$ s and $n 0 \mathrm{~s}$. We refer to any word in the alphabet $\{0,1\}$ with the property that the number of $1 \mathrm{~s}$ is $m$ times the number of $0 \mathrm{~s}$ as $m$-balanced. We denote the length of a word $w$ by $|w|$. For the partition $\lambda=(2,2,1,1,0) \in Y_{1,5}$ depicted in Figure 1, we have that $w_{\lambda}=0001101101$

We now associate a weight $\operatorname{wt}(w)$ with any $m$-balanced word $w=w_{1} \ldots w_{(m+1) n}$ :

$$
\operatorname{wt}(w):=\sum_{w_{i}=1} i
$$

This given, define

$$
B_{m, n}:=\left\{w \in\{0,1\}^{(m+1) n} \mid w m \text {-balanced, } w_{1}=0, \operatorname{wt}(w)=-1(\bmod n)\right\} .
$$

One may check that $w_{(2,2,1,1,0)}=0001101101$ belongs to $B_{1,5}$ as its weight equals 34 , which indeed is $-1(\bmod 5)$.

Before establishing a couple of lemmas that emphasize the importance of $B_{m, n}$, we need some notions from the combinatorics on words [12]. Given a word $w=w_{1} \cdots w_{k}$, define $\operatorname{rotate}(w):=w_{2} \cdots w_{k} w_{1}$. Clearly, $\operatorname{rotate}^{k}(w)=w$. We say that $w$ is primitive (or aperiodic) if no proper cyclic rotation of $w$ coincides with $w$. In other words, $w=w_{1} \ldots w_{k}$ is primitive if $\operatorname{rotate}^{j}(w) \neq w$ for $1 \leqslant j \leqslant k-1$. For instance, 0101 is not primitive while 0011 is. If $w$ is not primitive, it may be written as $w=\widehat{w}^{k / d}$ for some primitive $\widehat{w}$ and $d$ a proper divisor of $k$ (that is, $d$ cannot equal $k$ ). We say that two words are conjugate if one is obtained as a cyclic rotation of the other. Observe that a conjugate of a primitive word is again primitive.

LEMma 2.2. Every $w \in B_{m, n}$ is primitive.

Proof. Towards establishing a contradiction, suppose $w$ is not primitive. Then $w=$ $\widehat{w}^{(m+1) n / d}$ where $\widehat{w}$ is primitive and $d$ is a proper divisor of $(m+1) n$. We claim that $\widehat{w}$ is $m$-balanced as well. Indeed, say $\widehat{w}$ possesses $r$ s. It must be that

$$
\frac{(m+1) n}{d} r=n
$$

which implies that $r=\frac{d}{m+1}$. Thus, the number of 1 s in $\widehat{w}$ is $\frac{m d}{m+1}$, implying that $\widehat{w}$ is $m$-balanced. Note in particular that $(m+1) \mid d$.

Suppose $\operatorname{wt}(\widehat{w})$ equals $M$. Then we have that

$$
\begin{aligned}
\text { wt }(w)= & M+\left(M+\frac{m d}{m+1} d\right)+\left(M+\frac{m d}{m+1} 2 d\right) \\
& +\cdots+\left(M+\frac{m d}{m+1}\left(\frac{(m+1) n}{d}-1\right) d\right) \\
(2) \quad= & \frac{(m+1) n}{d} M+\frac{m d^{2}}{m+1}\left(\begin{array}{c}
\frac{(m+1) n}{d} \\
2
\end{array}\right),
\end{aligned}
$$

which, modulo $n$, translates to the equality

$$
\operatorname{wt}(w)=\frac{(m+1) n}{d} M-\frac{m n d}{2}(\bmod n) .
$$

Since $(m+1) \mid d$, we know that $m d$ is even. Thus $(3)$ simplifies to

$$
\mathrm{wt}(w)=\frac{(m+1) n}{d} M(\bmod n) .
$$


Since $w \in B_{m, n}$, we know that $\operatorname{wt}(w)=-1(\bmod n)$. This in conjunction with $(4)$ implies that $M$ satisfies

$$
\frac{(m+1) n}{d} M=-1(\bmod n) .
$$

Writing $(m+1) n / d$ as $\frac{n}{d /(m+1)}$, and recalling that $d$ is a proper divisor of $(m+1) n$, we conclude that $\operatorname{GCD}\left(\frac{(m+1) n}{d}, n\right) \geqslant 2$. In particular, (5) has no solutions, and we have established that $w$ is primitive.

We are now ready to relate $B_{m, n}$ to $Y_{m, n}$.

LEMMA 2.3. $B_{m, n}$ and $Y_{m, n}$ have the same cardinality.

Proof. We claim that the correspondence $\lambda \mapsto w_{\lambda}$ is a bijection from $Y_{m, n}$ to $B_{m, n}$. To this end, we first show that $w_{\lambda} \in B_{m, n}$. For convenience, set $w=w_{1} \ldots w_{(m+1) n}:=$ $w_{\lambda}$. It is immediate that $w_{1}=0$ as $\lambda_{1} \leqslant n-1$, and that $w$ is $m$-balanced. Thus we need to check that $\operatorname{wt}(w)=-1(\bmod n)$. It is easy to see that $w_{j}=1$ if and only if $j=n-\lambda_{i}+i$ for some (unique) $i$. Thus we obtain

$$
\operatorname{wt}(w)=\sum_{1 \leqslant i \leqslant N}\left(n-\lambda_{i}+i\right)=N n+\left(\begin{array}{c}
N+1 \\
2
\end{array}\right)-|\lambda| \text {. }
$$

Since $N=m n$ and $|\lambda|=(N-2)(n-1) / 2(\bmod n)$, we have

$$
\operatorname{wt}(w)=N n+\left(\begin{array}{c}
m \\
2
\end{array}\right) n^{2}+m n+n-1 .
$$

Thus we conclude that $\operatorname{wt}(w)=-1(\bmod n)$, and therefore $w \in B_{m, n}$.

It is clear that this correspondence is an injection from $Y_{m, n}$ to $B_{m, n}$. That this is a bijection follows because this correspondence is easily reversible, and one may obtain a partition for every word in $B_{m, n}$. That this partition belongs to $Y_{m, n}$ follows by reading the earlier string of equalities backwards.

We use this correspondence to obtain a "closed form" for $\left|Y_{m, n}\right|$.

COROLlaRY 2.4. The number of orbits of $\mathcal{C}_{m, n}$ under $\tau_{m, n}$ equals the number of $N$ element subsets of $[(m+1) n-1]:=\{1, \ldots,(m+1) n-1\}$ whose subset sum equals $-1(\bmod n)$. More explicitly, we have

$$
\left|Y_{m, n}\right|=\frac{1}{n} \sum_{d \mid n}(-1)^{m(n+d)} \mu(n / d)\left(\begin{array}{c}
(m+1) d-1 \\
m d
\end{array}\right) .
$$

Proof. Given $w=w_{1} \ldots w_{(m+1) n} \in B_{m, n}$, associate an $N$-element subset $S_{w}$ of $[(m+$ 1) $n-1]$ by

$$
S_{w}=\left\{j-1 \mid w_{j}=1\right\}
$$

It is clear that the sum of elements in $S_{w}$ is $-1(\bmod n)$, and that this correspondence sets up a bijection between $B_{m, n}$ and $N$-element subsets of $[(m+1) n-1]$ with subset sum equal to $-1(\bmod n)$. It remains to count such subsets, and we appeal to $[6$, Theorem 1.1] to this end. Setting $u=m+1, v=m$ and $c=n-1$ in loc. cit. implies that

$$
\begin{aligned}
B_{m, n} & =\frac{1}{(m+1) n} \sum_{d \mid n}(-1)^{m(n+d)} \mu(n / d)\left(\begin{array}{c}
(m+1) d \\
m d
\end{array}\right) \\
& =\frac{1}{n} \sum_{d \mid n}(-1)^{m(n+d)} \mu(n / d)\left(\begin{array}{c}
(m+1) d-1 \\
m d
\end{array}\right),
\end{aligned}
$$

which completes the proof. 


\section{The $h$-Positivity of $\operatorname{Frob}\left(\widehat{\tau}_{m, n}\right)$}

In this section we establish the $h$-positivity of the action $\widehat{\tau}_{m, n}$ on $\widehat{\mathcal{C}}_{m, n}$. We do this by determining representatives of equivalence classes in $\widehat{C}_{m, n}$ that carry the natural action of $S_{N}$ which permutes coordinates. As remarked earlier, in [11], we made a choice of representatives with the property that the first $N-1$ coordinates give a rational parking function. Unfortunately, this choice does not give rise to an $S_{N^{-}}$ stable set typically.

LEMMA 3.1. For every $\lambda \in Y_{m, n}$, the cardinality of the set $\left\{\right.$ sort $\circ \operatorname{shift}^{j}(\lambda) \mid 0 \leqslant j \leqslant$ $n-1\}$ is $n$.

Proof. We exploit a nice connection between rotations of $m$-balanced words and the shift map applied to elements of $Y_{m, n}$. Consider the lattice path $L_{\lambda}$ associated to $\lambda \in Y_{m, n}$, as well as its infinite extension $L_{\lambda}^{\infty}$. Label the $(m+1) n$ steps of $L_{\lambda}$ with integers 1 through $(m+1) n$ going right to left.

Let the labels of the horizontal steps be $a_{0}<a_{1}<\cdots<a_{n-1}$. This given, here is how one may compute sort $\circ \operatorname{shift}^{j}(\lambda)$ for $0 \leqslant j \leqslant n-1$. Consider the subpath of $L_{\lambda}^{\infty}$ of length $(m+1) n$ that begins with the horizontal step labeled $a_{j}$ and proceeds northwest. This subpath may be treated as $L_{\tilde{\lambda}}$ (after a potential translation) for a unique partition $\tilde{\lambda} \in Y_{m, n}$. It is not hard to see that $\widetilde{\lambda}=\operatorname{sort} \circ \operatorname{shift}^{j}(\lambda)$ by realizing that sort $\circ$ shift is essentially a "rotation" given that $\lambda$ is weakly decreasing from left to right. For instance, consider Figure 2 where the partition sort $\circ \operatorname{shift}^{3}(\lambda)$ is depicted by the parallel-hatched region in the translated $5 \times 5$ box for $\lambda=(2,2,1,1,0)$.

From the fact that $L_{\lambda}^{\infty}$ is built by periodically repeating $L_{\lambda}$, it follows that

$$
w_{\tilde{\lambda}}=\operatorname{rotate}^{a_{j}-1}\left(w_{\lambda}\right) .
$$

Now, recall that $w_{\lambda}$ is primitive by Lemma 2.2 , and thus $w_{\tilde{\lambda}}$ and $w_{\lambda}$ are distinct. This in turn implies that $\tilde{\lambda}$ and $\lambda$ are distinct as well. It follows that the set \{sort $\left.\circ \operatorname{shift}^{j}(\lambda) \mid 0 \leqslant j \leqslant n-1\right\}$ indeed has $n$ elements, one for each conjugate of $w_{\lambda}$ beginning with a 0 .

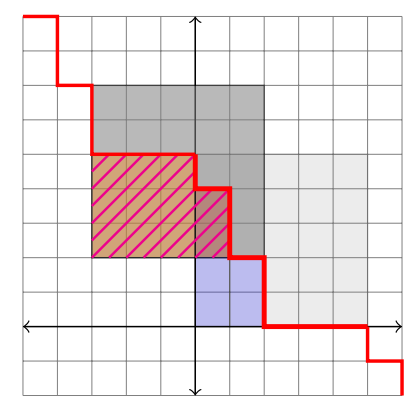

FigurE 2. The partition sort $\circ \operatorname{shift}^{3}(\lambda)$ realized by $L_{\widetilde{\lambda}}$ where $\lambda=(2,2,1,1,0)$.

We now exploit this lemma to extract a set of representatives for the equivalence classes in $\widehat{\mathcal{C}}_{m, n}$ that is $S_{N}$-stable.

Given $w \in B_{m, n}$, we denote the associated partition $\lambda$ satisfying $w_{\lambda}=w$ as $\lambda_{w}$. Recall that the conjugacy class of $w$ consists of all $(m+1) n$ cyclic rotations thereof. Amongst these cyclic rotations, there is a unique lexicographically smallest word, where the order is inherited by declaring $0<1$. Such a word is known as a Lyndon 
word. Observe the crucial fact that Lyndon word in the conjugacy class of $w \in B_{m, n}$ must itself belong to $B_{m, n}$, as it must begin with a 0 and cyclic rotations preserve weights. We denote the set of Lyndon words in $B_{m, n}$ by $B_{m, n}^{L}$.

Example 3.2. Consider $\mathcal{C}_{1,4}$. The 8 partitions in $Y_{1,4}$ (commas and parentheses suppressed) are given below. Those in the same column are obtained by applying sortoshift ${ }^{j}$ for $0 \leqslant j \leqslant 3$ to the highlighted partition.

21001110
32112221
32203332
33103000

It can be checked that the words $w_{\lambda}$ corresponding to the highlighted partitions are indeed Lyndon, and thus

$$
B_{1,4}^{L}=\{00101011,00011101\}
$$

More importantly, since elements of $Y_{1,4}$ index $S_{4}$-orbits of $\mathcal{C}_{1,4}$, and $\widehat{\mathcal{C}}_{1,4}$ is obtained by identifying elements of $\mathcal{C}_{1,4}$ up to shifts, it follows that the $S_{4}$-orbits of 2100 and 1110 generate a system of representatives for equivalence classes in $\widehat{\mathcal{C}}_{1,4}$. This is the underlying idea of what follows.

Let $\mathcal{O}_{\lambda_{w}}$ denote the $S_{N^{-}}$orbit of $\lambda_{w}$ for $w \in B_{m, n}$. We claim that the set of elements of $\mathcal{C}_{m, n}$ that belong to the orbit of $\lambda_{w}$ for a Lyndon word $w \in B_{m, n}$ gives a complete set of representatives for equivalence classes in $\widehat{\mathcal{C}}_{m, n}$. Indeed, we know that

$$
\mathcal{C}_{m, n}=\coprod_{w \in B_{m, n}} \mathcal{O}_{\lambda_{w}},
$$

and by invoking Lemma 3.1 to rewrite the right-hand side of (10) we get that

$$
\begin{aligned}
\mathcal{C}_{m, n} & =\coprod_{w \in B_{m, n}^{L}} 0 \leqslant j \leqslant n-1 \\
& =\coprod_{w \in B_{m, n}^{L}} \bigcup_{\text {sortoshift }^{j}\left(\lambda_{w}\right)} \coprod_{j \leqslant n-1} \mathcal{O}_{\text {shift }^{j}\left(\lambda_{w}\right) .}
\end{aligned}
$$

Since shift commutes with the action of $S_{N}$, we can rewrite (11) as

$$
\mathcal{C}_{m, n}=\coprod_{w \in B_{m, n}^{L}} \coprod_{0 \leqslant j \leqslant n-1} \operatorname{shift}^{j}\left(\mathcal{O}_{\lambda_{w}}\right),
$$

where we interpret $\operatorname{shift}^{j}\left(\mathcal{O}_{\lambda_{w}}\right)$ as the set obtained by applying shift ${ }^{j}$ to all elements in $\mathcal{O}_{\lambda_{w}}$. Since $\widehat{\mathcal{C}}_{m, n}$ is obtained by identifying sequences in $\mathcal{C}_{m, n}$ up to shifts, (12) tells us that we may identify $\widehat{\mathcal{C}}_{m, n}$ with $\coprod_{w \in B_{m, n}^{L}} \mathcal{O}_{\lambda_{w}}$, and thus $\widehat{\tau}_{m, n}$ is indeed the permutation action on the latter set. We are now ready to record an immediate consequence of this argument.

TheOREM 3.3. To each $w \in B_{m, n}^{L}$, associate a sequence $\mathrm{c}(w)$ that records the lengths of the maximal runs of $1 s$ in $w$. Then we have that

$$
\operatorname{Frob}\left(\widehat{\tau}_{m, n}\right)=\sum_{w \in B_{m, n}^{L}} h_{\text {sort }(\mathrm{c}(w))}
$$

In particular, we have that the number of orbits of $\widehat{\mathcal{C}}_{m, n}$ under $\widehat{\tau}_{m, n}$ is given by

$$
\left|B_{m, n}^{L}\right|=\frac{\left|Y_{m, n}\right|}{n}=\frac{1}{n^{2}} \sum_{d \mid n}(-1)^{m(n+d)} \mu(n / d)\left(\begin{array}{c}
(m+1) d-1 \\
m d
\end{array}\right) .
$$


Recall that in [11, Theorem 6.1], the number of orbits was computed by way of explicit character values. See also [17, Section 5] for a topological interpretation for the numbers $\left|B_{m, n}^{L}\right|$.

EXAmple 3.4. Consider $m=2$ and $n=3$. The three Lyndon words in $B_{m, n}^{L}$ are

$$
001011111001111011010110111 .
$$

The corresponding sort $(\mathrm{c}(w))$ are 51,42 , and 321. Theorem 3.3 implies that

$$
\operatorname{Frob}\left(\widehat{\tau}_{m, n}\right)=h_{51}+h_{42}+h_{321} \text {. }
$$

\section{THE TRIMMED STANDARD PERMUTAHEDRON}

In this section we focus on the case $m=1$, or equivalently, $N=n$. Hence we suppress the $m$ from all notions introduced earlier. Our goal is to establish Theorem 1.1 stated in the introduction.

Recall that given $\lambda:=\left(\lambda_{1} \geqslant \cdots \geqslant \lambda_{n}\right) \in \mathbb{Z}^{n}$, we let $P_{\lambda}$ denote the polytope in $\mathbb{R}^{n}$ defined by considering the convex hull of the $S_{n}$ orbit of $\lambda$. The $P_{\lambda}$ 's are referred to as usual permutahedra. The set of lattice points in $P_{\lambda}$, that is $\operatorname{Lat}\left(P_{\lambda}\right)$, is clearly $S_{n}$-stable, and one obtains a natural class of $S_{n}$-modules in this manner. Furthermore, since the stabilizer of any point in Lat $\left(P_{\lambda}\right)$ is a Young subgroup of $S_{n}$, we are guaranteed $h$-positivity of the associated Frobenius characteristic. It is a priori unclear whether these modules are of any value other than the intrinsic one. In what follows, we discuss the case of a special permutahedron, and show that its set of lattice points indexes the orbits of $\widehat{\mathcal{C}}_{n}$ under the action of $\widehat{\tau}_{n}$.

Fix $n \geqslant 2$. Let $\delta_{n}=(n-2, \ldots, 1,0,0) \in \mathbb{N}^{n}$. We are interested in the $S_{n}$ action on $\operatorname{Lat}\left(P_{\delta_{n}}\right)$, which we denote by $\gamma_{n}$. The reason behind this is the equality

$$
\left|\operatorname{Lat}\left(P_{\delta_{n}}\right)\right|=n^{n-2} \text {. }
$$

We briefly explain how to arrive at this equality through work of Postnikov [14], leaving it to the reader to check loc. cit. for further details. Consider the standard permutahedron $P_{(n-1, \ldots, 1,0)}$. The Minkowski difference of the standard permutahedron with the standard simplex $P_{(1,0, \ldots, 0)}$ is another permutahedron $P_{(n-2, n-2, \ldots, 1,0)}$. Following [14, Definition 11.2], we refer to the latter as the trimmed standard permutahedron. By [14, Corollary 11.5], we have that

$$
\left|\operatorname{Lat}\left(P_{(n-2, n-2, \ldots, 1,0)}\right)\right|=n^{n-2} .
$$

That this is equivalent to the equality in (13) is because translating $P_{(n-2, n-2, \ldots, 1,0)}$ by $(n-2, \ldots, n-2)$ followed by negating all coordinates maps it to $P_{\delta_{n}}$. It is also clear that this map is $S_{n}$-equivariant, so the $S_{n}$ action on $P_{\delta_{n}}$ is isomorphic to that on $P_{(n-2, n-2, \ldots, 1,0)}$, which also explains the title of this section.

The right-hand side of (13) naturally raises the question whether this $S_{n}$-action is related to the parking function representation. Indeed, as we shall soon establish, upon restricting this action to $S_{n-1}$ we recover the parking function representation.

EXAmple 4.1. Suppose $n=4$. Then $\delta_{n}=(2,1,0,0)$. The 16 elements in $\operatorname{Lat}\left(P_{\delta_{n}}\right)$ are given by the $S_{4}$-orbits of $(2,1,0,0)$ and $(1,1,1,0)$; compare these two orbit representatives with those encountered in Example 3.2. It follows that

$$
\operatorname{Frob}\left(\gamma_{4}\right)=h_{211}+h_{31}
$$

which upon restricting to $S_{3}$ gives $h_{3}+3 h_{21}+h_{111}$. This last expression is the Frobenius characteristic of the $S_{3}$ action on parking functions of length 3 . An alternative perspective is by projecting $P_{\delta_{4}}$ onto the hyperplane $x_{4}=0$ in $\mathbb{R}^{4}$ and realizing that $S_{3}$ 
acts on the lattice points of the resulting polytope. The $h_{111}$ term comes from the orbit of the point $(2,1,0)$, the three $h_{21}$ terms come from the orbits of $(1,0,0),(2,0,0)$, and $(1,1,0)$ respectively, and the $h_{3}$ term comes from the orbit of $(1,1,1)$.

To establish that $\gamma_{n}$ is isomorphic to $\tau_{n}$, we identify representatives of the equivalence classes in $\widehat{\mathcal{C}_{n}}$ that belong to $\operatorname{Lat}\left(P_{\delta_{n}}\right)$. Clearly, the $S_{n}$ action on $\operatorname{Lat}\left(P_{\delta_{n}}\right)$ has orbits indexed by elements of $\operatorname{Par}_{\leqslant} \delta_{n}$, which we defined to be the set of lattice points $\left(\lambda_{1}, \ldots, \lambda_{n}\right)$ in $P_{\delta_{n}}$ such that $\lambda_{1} \geqslant \cdots \geqslant \lambda_{n}$. Put differently, Par $\leqslant \delta_{n}$ consists of all partitions of size $\left(\begin{array}{c}n-1 \\ 2\end{array}\right)$ and length at most $n$ that are dominated by $\delta_{n}$ [16]. Note that all elements in $\operatorname{Par}_{\leqslant \delta_{n}}$ do indeed belong to $\mathcal{C}_{n}$.

LEMMA 4.2. Given $\lambda=\left(\lambda_{1}, \ldots, \lambda_{n}\right) \in \operatorname{Par}_{\leqslant \delta_{n}}$, no element in the set $\left\{\right.$ sort $\circ \operatorname{shift}^{j}(\lambda) \mid$ $1 \leqslant j \leqslant n-1\}$ belongs to $\operatorname{Par}_{\leqslant} \delta_{n}$.

Proof. We employ the lattice paths $L_{\lambda}$ and $L_{\lambda}^{\infty}$ defined earlier. Label the horizontal steps in $L_{\lambda}$ with integers 0 through $n-1$ going right to left. Fix a $j$ such that $0 \leqslant j \leqslant n-1$. Consider the fragment $L^{\prime}$ of $L_{\lambda}^{\infty}$ of length $2 n$ that starts with the horizontal step labeled $j$ and proceeds northwest. As discussed before, the partition determined by $L^{\prime}$ (in the bottom left corner of the $n \times n$ box this path naturally lives in) is sort $\circ \operatorname{shift}^{j}(\lambda)$. If we let $i$ denote the number of vertical steps in $L$ preceding the horizontal step labeled $j$, then we have

$$
\mid \text { sort } \circ \operatorname{shift}^{j}(\lambda)|=| \lambda \mid+(j-i) n \text {. }
$$

Now suppose there exists $j \neq 0$ such that $\mid$ sort $\circ \operatorname{shift}^{j}(\lambda) \mid \in \operatorname{Par}_{\leqslant} \delta_{n}$. From (15) it follows that $j=i$. Thus, the horizontal step labeled $j$ must touch the diagonal $x+y=n$. Let $\nu=\left(\nu_{1}, \ldots, \nu_{n-j}\right)$ be the partition determined by the subpath of $L$ restricted to the $(n-j) \times(n-j)$ box in the top left. Let $\mu=\left(\mu_{1}, \ldots, \mu_{j}\right)$ be the partition determined by the subpath of $L$ restricted to the $j \times j$ box in the bottom right. Now, observe that

$$
\begin{aligned}
\lambda & =\left(n-j+\mu_{1}, n-j+\mu_{2}, \ldots, n-j+\mu_{j}, \nu_{1}, \ldots, \nu_{n-j}\right) \\
\text { sort o } \operatorname{shift}^{j}(\lambda) & =\left(j+\nu_{1}, j+\nu_{2}, \ldots, j+\nu_{n-j}, \mu_{1}, \ldots, \mu_{j}\right) .
\end{aligned}
$$

Since $\lambda$ is dominated by $\delta_{n}$, we know that

$$
\sum_{k=1}^{j}\left(n-j+\mu_{k}\right) \leqslant \sum_{k=1}^{j}(n-j+k-2)=j(n-j-1)+\left(\begin{array}{l}
j \\
2
\end{array}\right) .
$$

Since the left-hand side is $|\lambda|-|\nu|=\left(\begin{array}{c}n-1 \\ 2\end{array}\right)-|\nu|$, we may rewrite the above inequality as

$$
|\nu| \geqslant\left(\begin{array}{c}
n-1 \\
2
\end{array}\right)-j(n-j-1)-\left(\begin{array}{l}
j \\
2
\end{array}\right)=\left(\begin{array}{c}
n-j-1 \\
2
\end{array}\right) .
$$

On the other hand, since our assumption is that sort $\circ \operatorname{shift}^{j}(\lambda)$ is also dominated by $\delta_{n}$, we obtain

$$
\sum_{k=1}^{n-j}\left(j+\nu_{k}\right) \leqslant \sum_{k=1}^{n-j}(j-2+k)=(n-j)(j-1)+\left(\begin{array}{c}
n-j \\
2
\end{array}\right) .
$$

This in turn may be rewritten as

$$
|\nu| \leqslant\left(\begin{array}{c}
n-j \\
2
\end{array}\right)-n+j=\left(\begin{array}{c}
n-j-1 \\
2
\end{array}\right)-1,
$$

which is in contradiction with the inequality in (19). 
To illustrate, consider $n=10$ and $\lambda=(7,5,5,5,4,4,2,2,2,0) \vdash 36$ as shown in Figure 3 . The path $L_{\lambda}$ is shown in the $10 \times 10$ box shaded light gray. The red line shows the diagonal $y+x=10$ and a horizontal step touching this diagonal gives a value $j$ such that sort $\circ \operatorname{shift}^{j}(\lambda) \vdash 36$. In the figure, we have chosen $j=6$. The path $L^{\prime}$ is shown in the $10 \times 10$ box with the darker shade of gray, and we see that sort $\circ \operatorname{shift}^{6}(\lambda)=(8,8,8,6,3,1,1,1,0,0)$. Note that $\nu=(2,2,2,0)$ is determined by the path in the intersection of the two shaded regions. Also $\mu=(3,1,1,1,0,0)$ is the partition determined by subpath of $L_{\lambda}$ by the $6 \times 6$ box in the bottom right. The reader may verify that $(8,8,8,6,3,1,1,1,0,0) \notin \operatorname{Par} \leqslant \delta_{10}$.

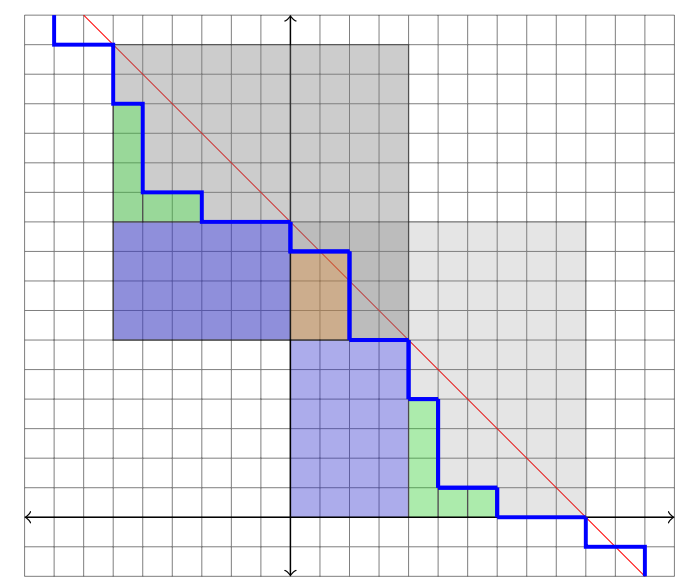

FiguRE 3. Path corresponding to $\lambda=(7,5,5,5,4,4,2,2,2,0)$.

We are now ready to establish our central result which essentially says that elements of $\operatorname{Lat}\left(P_{\delta_{n}}\right)$ form a complete set of representatives for equivalence classes in $\widehat{\mathcal{C}_{n}}$.

THEOREM 4.3. The representation $\gamma_{n}$ obtained by the $S_{n}$ action on $\operatorname{Lat}\left(P_{\delta_{n}}\right)$ is isomorphic to the representation $\widehat{\tau}_{n}$ obtained by the $S_{n}$ action on $\widehat{\mathcal{C}}_{n}$. Furthermore, the restriction of $\gamma_{n}$ to $S_{n-1}$ is $\operatorname{Park}_{n-1}$. The explicit $h$-expansion of $\operatorname{Frob}\left(\gamma_{n}\right)$ may be obtained as follows: Suppose mult $(\lambda)$ denotes the partition recording the multiplicities of each part in $\lambda$ for $\lambda \in \operatorname{Par}_{\leqslant \delta_{n}}$ (recall we are allowing 0 to be a part as well). Then

$$
\operatorname{Frob}\left(\gamma_{n}\right)=\operatorname{Frob}\left(\widehat{\tau}_{n}\right)=\sum_{\lambda \in \operatorname{Par}_{\leqslant} \delta_{n}} h_{\operatorname{mult}(\lambda)} .
$$

Proof. Note that each element in $\operatorname{Lat}\left(P_{\delta_{n}}\right)$ belongs to $\mathcal{C}_{n}$, and hence is a representative of some equivalence class in $\widehat{\mathcal{C}}_{n}$. Define $\varphi: \operatorname{Lat}\left(P_{\delta_{n}}\right) \rightarrow \widehat{\mathcal{C}}_{n}$ sending an element to the equivalence class in $\widehat{\mathcal{C}}_{n}$ that contains it. We claim that $\varphi$ is an $S_{n}$-equivariant bijection. The $S_{n}$-equivariance is immediate and so we focus on establishing bijectivity. Since $\left|\operatorname{Lat}\left(P_{\delta_{n}}\right)\right|=\left|\widehat{\mathcal{C}}_{n}\right|=n^{n-2}$, it suffices to show that $\varphi$ is an injection.

For $\lambda \in \operatorname{Par}_{\leqslant}$, let $\mathcal{O}_{\lambda}$ denote the $S_{n}$-orbit of $\lambda$. Then we know that

$$
\operatorname{Lat}\left(P_{\delta_{n}}\right)=\coprod_{\lambda \in \operatorname{Par}_{\leqslant} \delta_{n}} \mathcal{O}_{\lambda} .
$$

By Lemma 4.2, we infer that for distinct partitions $\gamma$ and $\mu$ in $\operatorname{Par}_{\leqslant} \delta_{n}$, we have $\varphi(\gamma) \neq \varphi(\mu)$. We now show that for distinct elements $x$ and $y$ in $\mathcal{O}_{\lambda}$ where $\lambda \in \operatorname{Par}_{\delta_{n}}$ is fixed, we have that $\varphi(x) \neq \varphi(y)$. Indeed suppose for the sake of contradiction that $\varphi(x)=\varphi(y)$. Since the $S_{n}$ action on $\mathcal{C}_{n}$ commutes with the shift map, we may assume without loss of generality that one of our two chosen elements, say $x$, equals $\lambda$. Suppose 
$y=\sigma \cdot \lambda$ for some $\sigma \in S_{n}$ that does not equal the identity. Now our hypothesis say that $\varphi(\lambda)=\varphi(\sigma \cdot \lambda)$, and hence we must have that $\sigma \cdot \lambda=\operatorname{shift}^{j}(\lambda)$ for some $1 \leqslant j \leqslant n-1$. But then sort $\circ \operatorname{shift}^{j}(\lambda)=\lambda \in \operatorname{Par}_{\leqslant} \delta_{n}$, which is a contradiction in view of Lemma 4.2. Finally we note that a similar argument tells us that if pick $x \in \mathcal{O}_{\lambda}$ and $y \in \mathcal{O}_{\mu}$ for $\lambda \neq \mu$ in $\operatorname{Par}_{\delta_{n}}$, then $\varphi(x) \neq \varphi(y)$.

In view of these facts just established and equation (22), we conclude that $\varphi$ is injective, and hence bijective. This discussion in conjunction with Remark 2.1 yields the statement of the theorem.

Taking [11, Theorem 3.1] into account, we get the following result.

Corollary 4.4. Let $\pi \in S_{n}$ have cycle type $\lambda=\left(\lambda_{1}, \ldots, \lambda_{\ell}\right)$ where $\lambda_{\ell}>0$. Set $d:=\operatorname{GCD}\left(\lambda_{1}, \ldots, \lambda_{\ell}\right)$. Then the number of lattice points in $\operatorname{Lat}\left(P_{\delta_{n}}\right)$ fixed by the action of $\pi$ is given by $f(d) n^{\ell-2}$ where

$$
f(d)= \begin{cases}1 & d=1 \\ 2 & d=2 \text { and } n=2(\bmod 4) \\ 0 & \text { otherwise. }\end{cases}
$$

Note that Corollary 1.2 now follows in view of [2, Theorem 1.2]. Is there a combinatorial proof of Corollary 4.4 which eschews character-theoretic computations?

We conclude with a couple of remarks.

REMARK 4.5. In private communication with the authors, S. Backman informed them that lattice points in the trimmed permutahedron $P_{\delta_{n}}$ may be interpreted as break divisors on the complete graph $K_{n}$, and the latter are in bijection with the set of spanning trees of $K_{n}$. Furthermore, in the divisor group of $K_{n}$, break divisors are in the same equivalence class as q-reduced divisors, which turn out be usual parking functions. We refer the reader to $[1,3,4]$ for more on these beautiful connections. By appealing to these (non-trivial) results, one could have bypassed our elementary Lemma 4.2 to arrive at Theorem 4.3.

REMARK 4.6. One could ask for a generalization of Theorem 4.3 when $m>1$. The following example shows that a naïve generalization may not work. Consider $m=2$ and $n=3$. Thus $c_{m, n}=1(\bmod 3)$. The orbits of $\mathcal{C}_{m, n}$ are indexed by the partitions

$$
\begin{aligned}
& 100000111100211000 \\
& 211111222211221110 \\
& 222220220000222100
\end{aligned}
$$

where the partitions in each column are obtained by applying sort $\circ \operatorname{shift}^{j}$ to the partition in the top row. Any three partitions, one from each column, index $S_{6}$-orbits for the action on $\widehat{\mathcal{C}}_{2,3}$. It is clear that in this instance that there is no way to pick three such partitions, all of the same size.

Acknowledgements. This material is based upon work supported by the Swedish Research Council under grant no. 2016-06596 while the authors were in residence at Institut Mittag-Leffler in Djursholm, Sweden during Spring 2020. We would like to thank the institute for its hospitality during our stay. The third author benefitted immensely from discussions with Darij Grinberg, Philippe Nadeau and Marino Romero. Thanks also to Brendon Rhoades, Spencer Backman and Steven Rayan for very helpful email correspondence(s). Finally we are very grateful to the anonymous referees for their comments that helped to improve exposition as well as the interesting questions raised. 


\section{REFERENCES}

[1] Yang An, Matthew Baker, Greg Kuperberg, and Farbod Shokrieh, Canonical representatives for divisor classes on tropical curves and the matrix-tree theorem, Forum Math. Sigma 2 (2014), Paper no. e24 (25 pages).

[2] Federico Ardila, Anna Schindler, and Andrés R. Vindas-Meléndez, The equivariant volumes of the permutahedron, Sém. Lothar. Combin. 82B (2020), Paper no. 16 (12 pages).

[3] Spencer Backman, Riemann-Roch theory for graph orientations, Adv. Math. 309 (2017), 655691.

[4] Matthew Baker and Serguei Norine, Riemann-Roch and Abel-Jacobi theory on a finite graph, Adv. Math. 215 (2007), no. 2, 766-788.

[5] Andrew Berget and Brendon Rhoades, Extending the parking space, J. Combin. Theory Ser. A 123 (2014), 43-56.

[6] Shane Chern, An extension of a formula of Jovovic, Integers 19 (2019), Paper no. A47 (7 pages).

[7] Giacomo d'Antonio and Giovanni Gaiffi, Symmetric group actions on the cohomology of configurations in $\mathbb{R}^{d}$, Atti Accad. Naz. Lincei Rend. Lincei Mat. Appl. 21 (2010), no. 3, 235-250.

[8] Nicholas Early and Victor Reiner, On configuration spaces and Whitehouse's lifts of the Eulerian representations, J. Pure Appl. Algebra 223 (2019), no. 10, 4524-4535.

[9] Giovanni Gaiffi, The actions of $S_{n+1}$ and $S_{n}$ on the cohomology ring of a Coxeter arrangement of type $A_{n-1}$, Manuscripta Math. 91 (1996), no. 1, 83-94.

[10] Mark D. Haiman, Conjectures on the quotient ring by diagonal invariants, J. Algebraic Combin. 3 (1994), no. 1, 17-76.

[11] Matjaž Konvalinka and Vasu Tewari, Some natural extensions of the parking space, J. Combin. Theory Ser. A 180 (2021), Paper no. 105394 (19 pages).

[12] M. Lothaire, Combinatorics on words, Cambridge Mathematical Library, Cambridge University Press, Cambridge, 1997, With a foreword by Roger Lyndon and a preface by Dominique Perrin, Corrected reprint of the 1983 original, with a new preface by Perrin.

[13] Olivier Mathieu, Hidden $\Sigma_{n+1}$-actions, Comm. Math. Phys. 176 (1996), no. 2, 467-474.

[14] Alexander Postnikov, Permutohedra, associahedra, and beyond, Int. Math. Res. Not. IMRN (2009), no. 6, 1026-1106.

[15] Alexander Postnikov and Boris Shapiro, Trees, parking functions, syzygies, and deformations of monomial ideals, Trans. Amer. Math. Soc. 356 (2004), no. 8, 3109-3142.

[16] Richard Rado, An inequality, J. London Math. Soc. 27 (1952), 1-6.

[17] Steven Rayan, Aspects of the topology and combinatorics of Higgs bundle moduli spaces, SIGMA Symmetry Integrability Geom. Methods Appl. 14 (2018), Paper No. 129.

[18] Richard P. Stanley, A zonotope associated with graphical degree sequences, in Applied geometry and discrete mathematics, DIMACS Ser. Discrete Math. Theoret. Comput. Sci., vol. 4, Amer. Math. Soc., Providence, RI, 1991, pp. 555-570.

[19] _ Enumerative combinatorics. Vol. 2, Cambridge Studies in Advanced Mathematics, vol. 62, Cambridge University Press, Cambridge, 1999, With a foreword by Gian-Carlo Rota and appendix 1 by Sergey Fomin.

[20] Sarah Whitehouse, The Eulerian representations of $\Sigma_{n}$ as restrictions of representations of $\Sigma_{n+1}$, J. Pure Appl. Algebra 115 (1997), no. 3, 309-320.

Matjaž Konvalinka, University of Ljubljana \& Institute of Mathematics, Physics and Mechanics, Department of Mathematics, Ljubljana, Slovenia

E-mail : matjaz.konvalinka@fmf.uni-lj.si

Robin Sulzgruber, York University, Department of Mathematics and Statistics, Toronto, Canada E-mail : rsulzg@yorku.ca

VASU Tewari, University of Hawaii at Manoa, Department of Mathematics, Honolulu, HI 96822, USA

E-mail : vvtewari@math.hawaii.edu 